\title{
Designing Interventions to Overcome Poor Numeracy and Improve Medication Adherence in Chronic Illness, Including HIV/Aids
}

\author{
John O. Moore • Edward W. Boyer • Steven Safren • \\ Gregory K. Robbins $\cdot$ Edwin D. Boudreaux • \\ Rochelle Rosen • Bruce Barton • Frank Moss
}

Published online: 1 April 2011

(C) American College of Medical Toxicology 2011

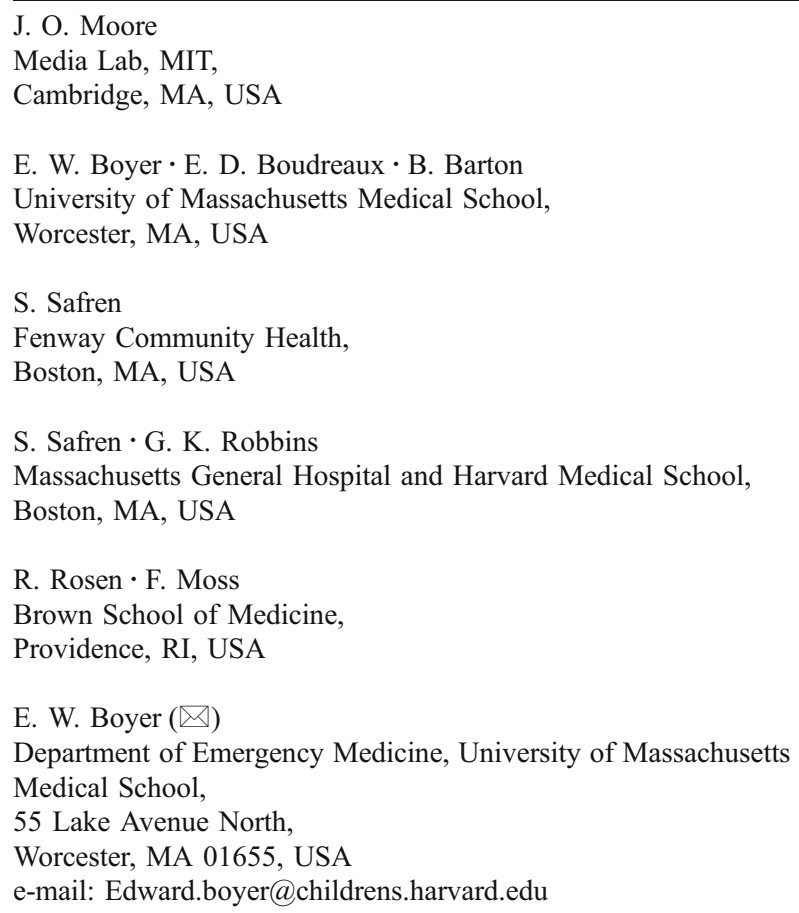

\begin{abstract}
Numeracy is an element of health literacy that refers to the ability to understand numerically related information. When applied to health behaviors, it describes the degree to which individuals have the capacity to access, process, interpret, and act on graphical and probabilistic health information. As a cognitive and functional skill, low numeracy correlates with poor outcomes in the management of chronic diseases; numeracy is therefore an essential component of patients' capacity to adhere to medication regimens. In this manuscript, we describe novel visual interventions to improve medication adherence in difficult, chronically ill populations. We have used personalized
\end{abstract}

graphical representations of plasma medication concentration and dynamic disease state simulation to overcome poor numeracy. These methods incorporate efficient, precise, and clear graphical data; cartographical techniques focused on judicious use of color intensities; and animation that increases engagement and accentuates information transfer.

Keywords Technology $\cdot \mathrm{mHealth} \cdot$ Medication adherence . HIV

\section{Introduction}

Numeracy is an element of health literacy that refers to the ability to understand numerically related information [1]. Although related to health literacy, numeracy is a distinct cognitive skill that involves proficiency in applying mathematical concepts and abilities to everyday tasks, often through comprehending approximate representations of numerical magnitude [2]. When applied to health behaviors, numeracy is the degree to which individuals have the capacity to access, process, interpret, and act on probabilistic health information $[2,3]$. As a cognitive and functional skill, numeracy is important in a patient's capacity to adhere to medication regimens, and low numeracy correlates with poor outcomes in the management of chronic diseases [2, 4]. Examples of medical instructions, statements, and information requiring various degrees of numeracy are presented in Table 1 [3].

Although the impact of health numeracy on patient selfmanagement and participatory decision making is increasingly recognized, most studies have focused on outcomes of poor numeracy rather than developed approaches to improve it [2]. 
Table 1 Examples of medical statements requiring health numeracy

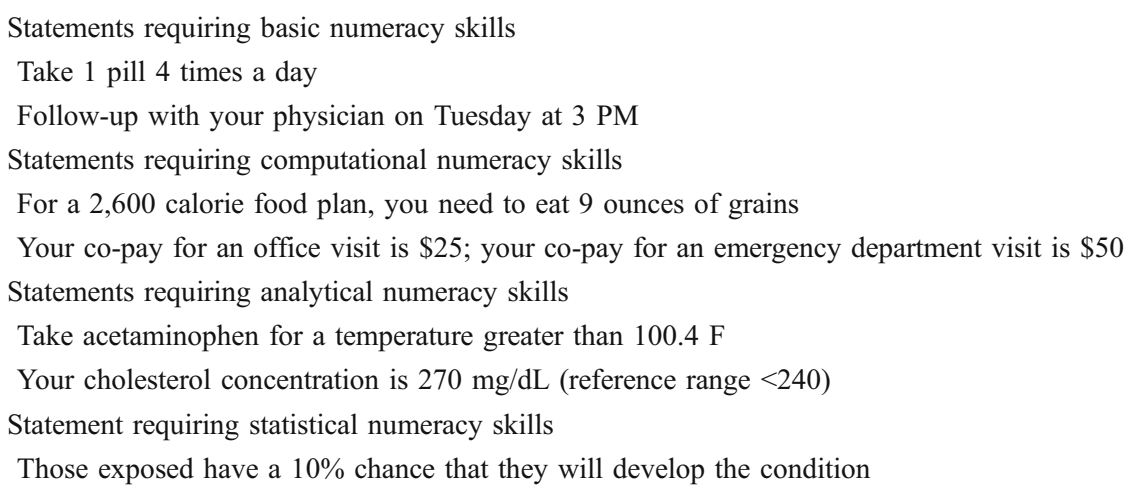

Take 1 pill 4 times a day

Follow-up with your physician on Tuesday at $3 \mathrm{PM}$

Statements requiring computational numeracy skills

For a 2,600 calorie food plan, you need to eat 9 ounces of grains

Your co-pay for an office visit is $\$ 25$; your co-pay for an emergency department visit is $\$ 50$

Statements requiring analytical numeracy skills

Take acetaminophen for a temperature greater than $100.4 \mathrm{~F}$

Your cholesterol concentration is $270 \mathrm{mg} / \mathrm{dL}$ (reference range <240)

Statement requiring statistical numeracy skills

Those exposed have a $10 \%$ chance that they will develop the condition

Numeracy is increasingly important because of the greater emphasis of health care policy on tapping the power of informed consumers to improve health outcomes and improve the efficiency of health care [1]. Unfortunately, not all consumers possess the aptitude, knowledge, or motivation necessary to utilize information related to health. Assigning greater responsibility to patients, therefore, may paradoxically exacerbate the disadvantages felt by those with poorer numeracy skills [5]. Disparities in health literacy and, in particular, numeracy would potentially lead to more rapid or greater functional declines in health in those with poorer skills. Because of the greater emphasis on patient self-management and participatory decision making, developing methods that counter poor numeracy is of heightened importance $[1,5]$.

Numeric skills that are often applied to decisions related to health care include evaluating the risks and benefits of treatment options, comparing short-term against long-term outcomes, and adhering to complex health regimens [1]. Because the presentation of data remains critical to supporting optimal health behaviors, the best practices for delivery of numeric health information often involve the effective visual display of quantitative data [1]. Minimizing the amount of cognitive effort in evaluating data, reducing inferences, and eliminating calculations minimize numeracy effects [1]. In this manuscript, we describe graphical methods to support medication adherence that are based upon realtime pharmacodynamic estimations of drug concentration and dynamic, personalized simulation of therapeutic medication on the disease being treated. When delivered via a mobile computing device such as a smartphone, this method has the potential to improve medication adherence in chronic disease states and, hence, improved health.

\section{Theoretical Basis for the Intervention}

The present approach to improve medication adherence intends to help individuals become well-informed (e.g., determine the outcome associated with medication adherence and nonadherence), motivated to act (e.g., take their medications according to schedule), and possess the behavioral skills needed to adhere to medications (e.g., the ability to take medications on a daily basis, but also to prepare for situations where medications may not normally be available such as travel). In this analysis, our approach is consistent with the information-motivation-behavioral (IMB) model that can be used to understand, predict, and promote chronic medication adherence [6]. The IMB model of adherence is based on a generalized IMB approach to understanding and promoting health behaviors that has been validated with over a decade's worth of correlational and experimental research $[6,7]$. The IMB model of adherence states that adherence-related information, motivation, and behavioral skills are fundamental determinants of adherence to highly active antiretroviral therapy (HAART) among HIV-infected individuals [6]. The IMB model suggests that HIV-positive persons who remain poorly informed about the impact of poor adherence to therapy, lack motivation to act, and do not have the behavioral skills to act are unlikely to adhere to antiretroviral therapies over time and experience limited health benefits [6]. Conversely, HIV-positive individuals who are wellinformed, motivated to take antiretroviral medications, and possess the behavioral skills needed to act effectively are more likely to enjoy better health through improved adherence to HAART regimens [6].

\section{Knowledge Necessary to Adhere to Chronic Medication Regimens, Including HAART}

Pharmaceutical adherence often requires that patients comply with instructions not only related to the timing of medication dose but also compatibility with food and requirements for fluid intake. For patients maintained on HAART, understanding medication regimens can be particularly difficult $[8,9]$. Some antiretroviral agents need to be taken with food and others between meals; some are taken once daily, whereas others are dosed three or more times a day [9]. Multidrug HAART regimens are among the 
most complicated open-ended therapies that have been devised, and high levels of adherence are crucial to longterm virologic control since lower adherence leads to the emergence of drug resistance. Understanding medication instruction, the timing of meals, and amount of fluid intake, in addition to the medication schedule, are therefore considered important for optimal outcomes. Even on its most basic level, the number of prescribed medications can be breathtaking: Formerly, HIV-positive patients ingested up to 15 medications per day [10]. The number of HAART medications, the complexity of the dosing schedule, drug side effects, as well as patients' perceptions of benefit from HAART adherence, have all been strongly linked with the degree of adherence [11].

\section{Graphical Interventions That Counteract Poor Numeracy}

Data graphics display measured quantities through the use of points, lines, symbols, words, shading, colors, and other visual formats to create an instrument for reasoning about numerical data [12]. For communicating information about trends and for minimizing inferences, well-designed graphics are often the simplest and most powerful [12]. The effectiveness of graphic data, however, requires that clarity, efficiency, and precision be used in the communication of complex and interconnected ideas [12]. In the context of medication adherence, these ideas include dose of medication(s), the time at which the medication should be retaken, and the consequences on health of adherence and nonadherence.

One potential way to communicate the importance of taking medications according to dosing schedule is to demonstrate the time-dependent change in plasma concentration determined by drug pharmacokinetics. By placing information relating drug adherence to outcome, a multivariate graphical presentation can emphasize that declining drug concentrations adversely affects health. Figure 1 demonstrates one such approach [13].

In this representation, red " $X$ "'s denote missed medication doses and green checks denote ingested medication [13]. Black lines represent estimated plasma concentration calculated from self-reported time of medication ingestion and pharmacokinetic parameters for that drug. Three horizontal regions of the image are important for conveying information related to potential outcome. Tinted regions, the color of which is matched to labels on patient's medication bottles, represent target serum concentrations for that drug. Hashed regions define subtherapeutic concentrations, while the white regions indicate minimal, even absent concentration of drug [13]. Although a plot of estimated time-dependent serum antiretroviral concentrations could be viewed as

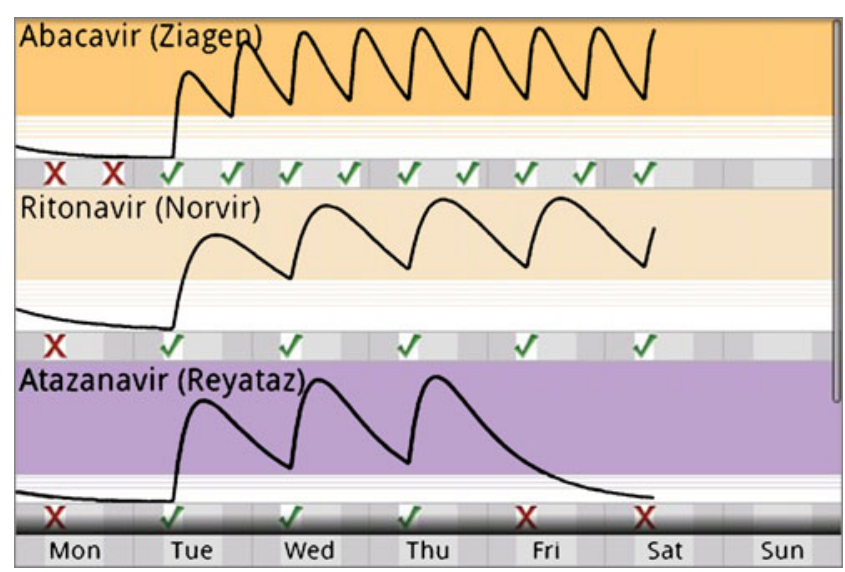

Fig. 1 Graphic representation showing the relationship between time of HAART ingestion and therapeutic serum drug concentration

confusing to users with poor numeracy, several graphical elements were designed to address this issue. First, the dynamic relationship between the act of ingesting the drug and the change in serum concentrations becomes personalized; moreover, transitioning data from an abstract thought to a visual image enables the user to more easily associate action with outcome. Additional cues solidify these relationships. For example, the presence of green checks is associated with a beneficial increase in the estimated drug concentration. Conversely, a detrimental decline in drug concentration follows a red " $\mathrm{X}$ " denoting a missed medication dose. Also, shaded and striped regions simplify the interpretation of the medication concentration at any point in time: Drug is present at target concentrations that are effective for the treatment of disease, at ineffective concentrations, or at an intermediate transition point. This visual image eliminates cluttering numerical scales that would dim the visual power of the graphic. This design is both precise and highly efficient; all elements of the graphic - plots, checks, and thresholdsadhere to the fundamental principles of good data graphics in that no non-data details are present [12].

The power of estimated serum concentration is even more clear when they are used to drive personalized, dynamic simulations of the actual effects of medications in the patient's body on the disease being treated (Fig. 2a-c). Several elements convey critical information. First, the number of $\mathrm{T}$ cells and viral particles are proportional to the patient's actual laboratory tests. Second, a segmented barrier surrounding each immune cell portrays the degree of protection from antiretroviral therapy. Each segment in the barrier represents a single medication; again, the color of the segment is matched to labels on the patient's medication bottles. If a medication is at target concentration, its segment is solid. The barrier is hashed if the concentration is suboptimal, and it is absent if the drug concentration is minimal. When all medication concentrations are at goal, $\mathrm{T}$ cells are fully protected. In this state, 


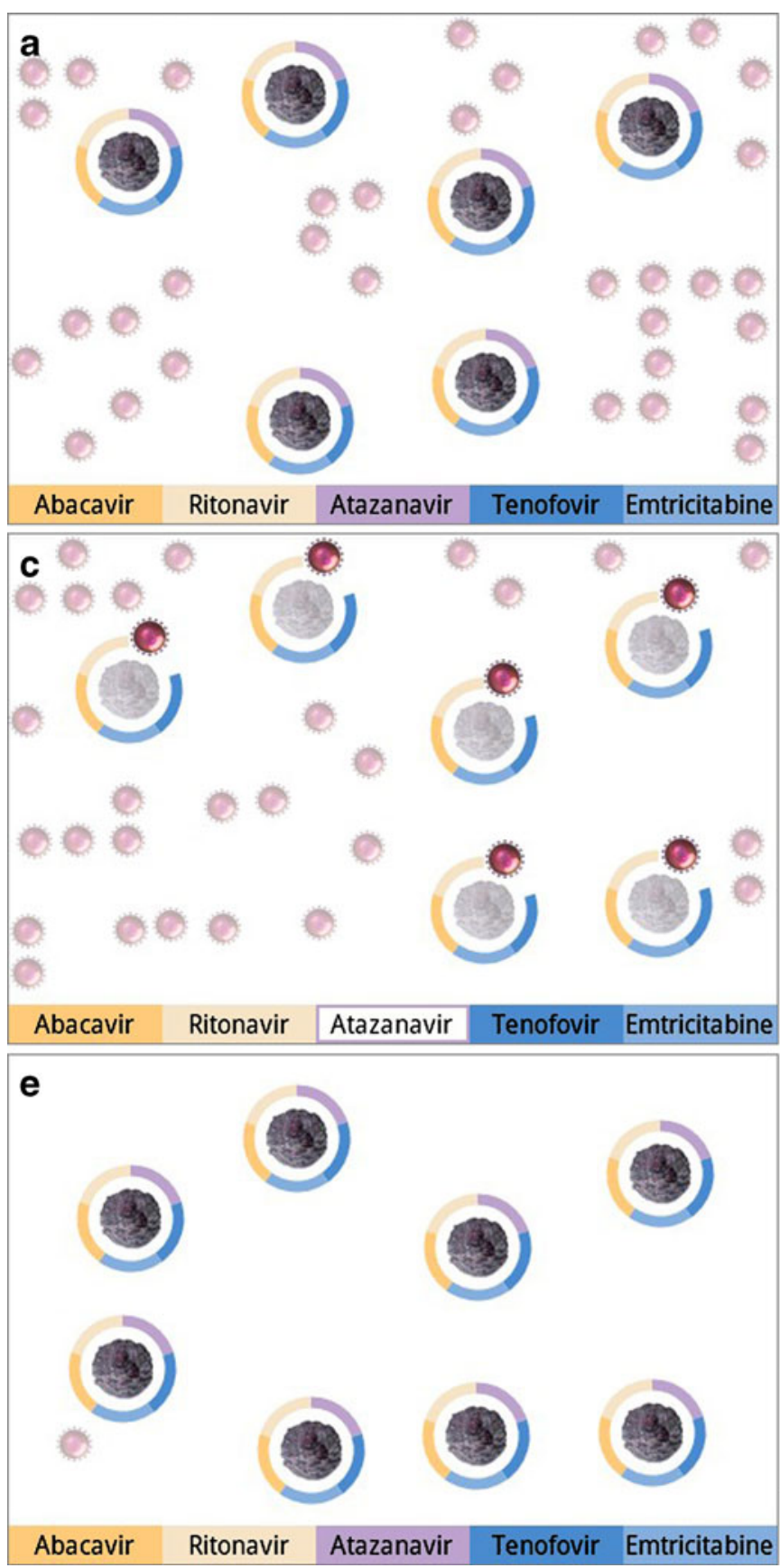

Fig. 2 a Good adherence to HAART regimen, b HIV simulation with one medication (atazanavir) in suboptimal concentration, $\mathbf{c}$ simulation of HIV status with one medication in minimal concentration, d

they have full intensity colors and move in smooth animations on the screen. Viral particles, because they have no $\mathrm{T}$ cells to attack, are smaller, are depicted in less vivid colors, and unmoving. When a medication concentration falls, the corresponding segment degrades to being hashed and then absent. Viruses, as they attack T cells, develop bolder colors and are animated in frenetic motion. $\mathrm{T}$ cells under attack become less vivid and lose motion.

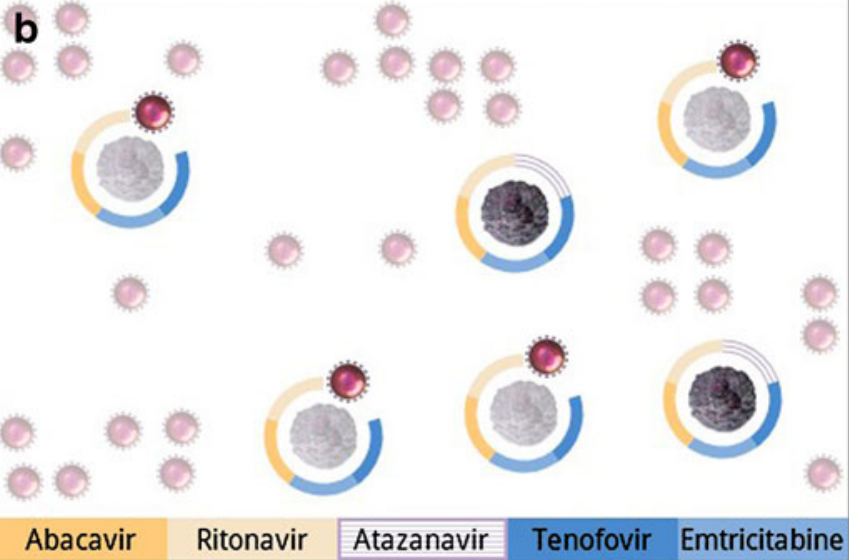

d

Q
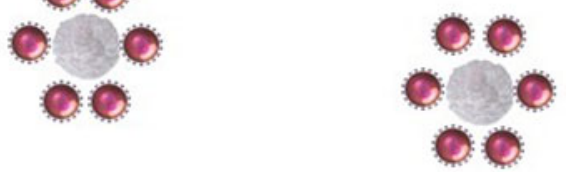

0
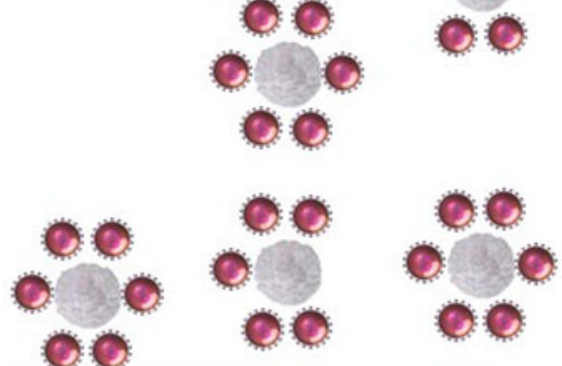

\begin{tabular}{lll|l|l|}
\hline Abacavir & Ritonavir Atazanavir & Tenofovir & Emtricitabine \\
\hline
\end{tabular}

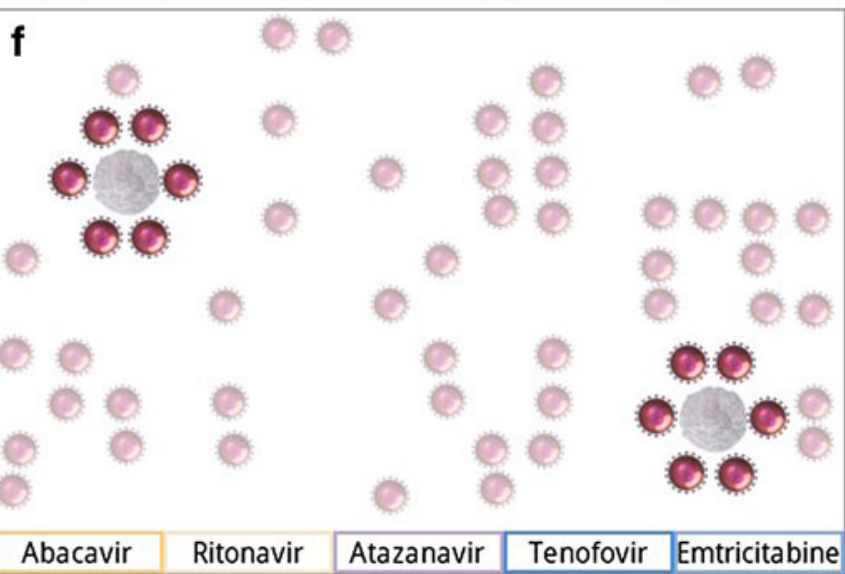

simulation of HIV with poor adherence, e simulation of HIV state after prolonged good adherence, $\mathbf{f}$ simulated HIV state after prolonged poor adherence

This graphical approach to behavioral intervention conveys information about HAART adherence in a way that eliminates the need for numerical data. First, the overall composition employs a concept known as "layering" of information [14]. In a first layer, the number of cells is a reflection of the patient's laboratory tests and longer-term adherence to HAART; the ratio of T cells to viral particles therefore changes over a period of weeks to months. An 
additional layer of information reflects the short-term effects of adherence. The barriers formed by antiretroviral medications and the dynamics of viruses attacking the $T$ cells can change in a matter of hours based upon real-time adherence data entered by the user. This combination of slowly changing context and real-time information is a powerful graphic image. Alone, each layer strongly establishes the relationship between taking medications and immune system protection; together, a stronger one. A second design effect involves altering the color of cells/ viruses. Using more intense colors connoting "more vivid equals more active" separates biological outcomes of HAART adherence. The effectiveness and elegance of small areas of saturated color for carrying information and emphasizing features is long recognized as a design secret of classical cartographers $[14,15]$. The third component, animation, synthesizes all of these elements to increase the forcefulness underlying the health message. Overall, this graphical approach accomplishes the visual reinforcement of a behavioral act by describing the scope of alternative outcomes, all of which pertain solely to the patient, are relevant to health, and directed toward the behavior of improving HAART adherence.

The utility of this approach has undergone preliminary assessment in a pilot study among study participants whose numeracy was presumed to be low $[13,16]$. In focus groups, $92 \%$ (11 of 12) of members were unable to spontaneously verbalize basic features of HIV infection or its treatment. After being shown the graphical interventions describe in Figs. 1 and 2, 92\% reported understanding the implications the HAART adherence. In a follow-up proof-of-concept study with four HIV+ patients over a 30-day period, the graphical intervention contributed to an improvement in HAART adherence. For three of four participants, the graphical interventions contributed to an improvement in adherence of up to $17 \%$ and helped the fourth to maintain optimal adherence of greater than $95 \%$. Importantly, the least compliant patients realized the greatest increases in HAART adherence. Self-reported adherence was validated by pill counts $[13,16]$. These data suggest the rapid, efficient conveyance of a mass of medical information in a way that supports improved health. Although this study targeted low numeracy patients, these results imply that graphical representations may be important in the modifying the health behaviors of those with average or even above average numeracy as well.

\section{Mobile Health Applications for Medication Adherence}

Generally speaking, the concept of mobile health ("mHealth") describes the use of mobile technologies as platforms for health research and healthcare delivery
[17]. mHealth applications include public health education, remote data collection, remote patient monitoring, and promotion of healthy behaviors [17]. While there is no panacea to the complex challenges of improving public health outcomes, mHealth-existing at the convergence of health and technology domains-provides a singular opportunity to bring about significant improvements [17]. Most mHealth strategies to improve medication adherence focused heavily on brief text message-based interventions, an approach that cannot counteract the poor numeracy and literacy common in patients with low rates of medication adherence. Unfortunately, text interventions not take full advantage of capabilities of smartphones nor the demographic surge toward use of these devices. Smartphones, being multimedia technologies, are highly suited to the delivery of graphical interventions, particularly those that are continually updated on the basis on real-time personalized data. Furthermore, it may not make sense to develop interventions that do not leverage the use of smartphone operating systems that are rapidly becoming normative.

\section{Conclusion}

Poor numeracy, considered to be a threat to an individual's perception and response to health, is associated with poor rates of medication adherence. Minimizing the amount of cognitive effort in evaluating data minimizes numeracy effects. Using principles of visual display of quantitative data, we have identified a smartphone-deliverable graphical intervention that supports HAART adherence. With displays that are clear, efficient, and evidential, well-designed graphical interventions have the capacity to communicate complex yet understandable health information to patients.

Conflicts of interest None.

\section{References}

1. Peters E, Hibbard J, Slovic P, Diekmann N (2007) Numeracy skill and the communication, comprehension, and use of risk-benefit information. Health Aff 26:741-745

2. Waldrop D, Jones D, Jayaweera D, Gonzalez P (2009) Gender differences in medication management capacity in HIV infection: the role of health literacy and numeracy. AIDS Behav 13:46-52

3. xGolbeck A, Ahlers-Schmidt C, Paschal A (2005) A definition and operational framework for health numeracy. Am J Prev Med 29:375-376

4. Kalichman S, Ramachandran B, Catz S (1999) Adherence to combination antiretroviral therapies in HIV patients of low health literacy. J Gen Intern Med 14:267-273 
5. Marcus E (2006) The silent epidemic - the health effects of illiteracy. NEJM 355:339-341

6. Fisher J, Fisher W, Amico K (2006) An information-motivationbehavioral skills model of adherence to antiretroviral therapy. Health Psychol 25:462-473

7. Fisher J, Fisher W, Amico K, Harman J (2003) A model for conceptualizing ART adherence behavior. In: South African AIDS Conference. Durban, South Africa.

8. Altice F, Friedland G (1998) The era of adherence to HIV therapy. Ann Intern Med 129:503-504

9. Wolf M, Davis T, Arozullah A, Penn R, Arnold C (2005) Relation between literacy and HIV treatment knowledge among patients on HAART regimens. AIDS Care 17:863-873

10. Murphy D, Roberts K, Martin D, Marelich W (2000) Barriers to antiretroviral adherence among HIV-infected adults. AIDS Patient Care STDs 14:47-58
11. Fogarty L, Roter D, Larson S, Levy R (2002) Patient adherence to HIV medication regimens: a review of published and abstract reports. Patient Educ Couns 46:93-108

12. Tufte E (1983) Visual display of quantitative information. Graphics, Cheshire

13. Moore J (2009) CollaboRhythm: new paradigms in doctorpatient interaction applied to HIV medication adherence. MIT, Cambridge

14. Tufte E (19909) Envisioning information. Graphics, Cheshire

15. Imhoff E (1965) Kartographische Gelandedarstellung. de Gruyter, Berlin

16. Moore J, Hardy H, Skoknik P, Moss F (2011) A collaborative awareness system for chronic disease medication adherence. J Am Med Informat Assoc (in press)

17. mHealth for Development, United Nations Foundation (2009) unf_mhealth_for_development_brochure.pdf. Accessed 22 Sep 2010 